\title{
Prognostic role of tumor-infiltrating lymphocytes in gastric cancer: a meta-analysis and experimental validation
}

\author{
Nana Zhang, Meng Cao, Yixin Duan, Haixia Bai, Xiang Li, Yili Wang
}

Institute of Cancer Research, School of Basic Medical Science, Xi'an Jiaotong University Xi'an, China

Submitted: 19 July 2017

Accepted: 15 February 2018

Arch Med Sci 2020; 16 (5): 1092-1103

DOI: https://doi.org/10.5114/aoms.2019.86101

Copyright (c) 2019 Termedia \& Banach

\section{Abstract}

Introduction: We performed a meta-analysis and an experimental validation to investigate the association between tumor infiltrating lymphocytes (TILS) and the outcome of gastric cancer (GC) patients to provide prognostic indicators for clinical practice.

Material and methods: The relative literature of TILs in tumor tissue from patients with gastric cancer was searched from PubMed, Embase, NIH databases, from April 2000 to 31 December 2016. Studies on the prognostic value of TILs as CD3+, CD4+, CD8+, GrB+, and FOXP3+ lymphocytes for GC were retrieved, and also the related references were traced as supplements. Independent screening documents, extracting information and evaluating quality were implemented independently by 2 evaluators according to the inclusion and exclusion criteria, which were then analyzed by meta-analysis using STATA version 12.0 software.

Results: The results indicated that high levels of intratumoral CD8+, CD3+ and CD4+ T cell infiltration were associated with better overall survival(OS) in gastric cancer patients, while high density of intratumoral FOXP $3+T$ cells was not closely associated with a worse outcome. Additionally, in our study, higher density of granzyme $\mathrm{B}+(\mathrm{GrB}+) \mathrm{T}$ cell infiltration indicated an optimistic prognosis, and infiltration of a larger number of general TILs also suggested a favorable prognosis by log-rank test analysis.

Conclusions: This meta-analysis clarified that high levels of CD8+, CD3+, and CD4+ T cell infiltration in tumor tissue showed better OS in GC patients, whereas high density of FOXP3 + T cell infiltration may not be recognized as a negative prognostic factor. These results may provide some useful prognostic indicators for clinical application in gastric cancer.

Key words: gastric cancer, meta-analysis, tumor infiltrating lymphocytes, prognostic indicators, outcome.

\section{Introduction}

Early in 1922, McCarty [1] had already brought up the concept of TILS, and considered the infiltration of lymphocytes into tumor tissue as an antitumor activity of the immune system. However, increasing evidence has indicated that the interactions between immune cells and cancers are amazingly complicated [2]. Recently, a bidirectional role of TILs within the tumor microenvironment in tumor progression has been confirmed.

\author{
Corresponding author: \\ Prof. Yili Wang MD, PhD \\ Institute of Cancer Research \\ Health Science Center of \\ Xi'an Jiaotong University \\ 76 YanTa XiLu, Xi'an \\ Shaanxi 710061, China \\ Phone: +8618691838129 \\ Fax: +862982655499 \\ E-mail: wangyili@mail.xjtu. \\ edu.cn
}


Namely, the TILs can not only suppress tumor growth by destroying cancer cells or inhibiting their outgrowth, but also promote tumor progression either by selecting for tumor cells that are more fit to survive in an immunocompetent host or by establishing conditions within the tumor microenvironment which could facilitate tumor outgrowth [3, 4], which may result from the fact that TILs are a heterogeneous population including $T$ cells with different functional phenotypes (occupying the main parts), B cells, and natural killer (NK) cells [5], which could interrupt the immune balance during cancer progression, because of their different physiological and pathological effects in the tumor microenvironment. Therefore, it is important to discuss the prognostic role of the main parts of TIL subsets separately. The correlation between TILs and clinical outcome have been investigated in many solid cancers [6-8]. Plenty of TILs related studies showed conflicting results in gastric cancer field, the prognostic value of TILs and/or TILs subsets was not yet determined $[9,10]$. As a result, the meta-analysis was conducted to identify the prognostic value of TILs and TILs subsets in GC patient stratified by infiltration sites. According to the lymphocyte functional phenotypes, we chose the following subtypes of $\mathrm{T}$ lymphocytes as our analysis objectives. CD3, a biomarker of $T$ lymphocytes, is expressed in almost all $T$ lymphocytes; CD8+ cytotoxic $T$ lymphocytes (CTLs) are directly capable of killing tumor cells; granzyme B (GrB) is the main effector molecule of activated cytotoxic $T$ lymphocytes (CTLs) and natural killer (NK) cells, and also is their specific activation marker; CD4+ T helper lymphocytes (Th) are a heterogeneous cytokine secreting class of T lymphocytes; $T$ helper type 1 lymphocytes (Th1) have a crucial role in activating CTLs; $\mathrm{T}$ helper type 2 lymphocytes stimulate humoral immunity and activate eosinophils; FOXP3+ regulatory cells (Tregs) play a critical role in immunotolerance and deficiency of anti-tumor immunity and some immune diseases [11-13].

\section{Material and methods}

\section{Literature search}

We searched the literature using the PubMed database, Embase, and NIH databases from April 2000 to 31 December 2016. The retrieval approach was adopted by combining the keywords with free words. Search terms included: immune cells, immune responses, tumor infiltrating lymphocytes, gastric, stomach, cardia or pyloric, and cancer, carcinoma, tumor or malignancy, and prognosis, prognostic or survival outcome. There were no other limitations in the database searching process. Additionally, all the reference lists of identified articles were also reviewed in order to find potential studies. Two authors were responsible for searching the comprehensive database and evaluating availability independently.

\section{Eligibility criteria}

Papers that were eligible for inclusion in this meta-analysis should meet the following criteria: (1) patients were diagnosed with gastric cancer; (2) prognostic roles of the general TILs or T lymphocyte subsets (including CD3+, CD8+, GrB+, CD4+, and FoxP3+ lymphocytes) were evaluated; (3) the TIL measurement must be detected in situ of tumor tissue by application of HE or immunohistochemistry; (4) the lymphocyte infiltration site of the study should be within the tumor tissue, such as tumor parenchyma, tumor stroma; (5) adequate data (HR, and its associated $95 \% \mathrm{Cl}$ ) were provided for the further analysis; (6) original article; when two or more studies were published by the same authors, the better quality paper was included. Exclusion criteria were as follows: (1) the studies were of insufficient data; (2) as small sample size would induce publication bias, studies with sample size $<50$ were excluded; (3) some styles of literature such as case reports, letters, reviews, conference abstracts and animal trials were excluded.

\section{Data extraction and quality assessment}

Two authors extracted the data independently from all eligible studies. Uncertainties were resolved through discussion. The following information was extracted from all included publications: author, country of study, publication year, number of patients, lymph node metastasis, tumor staging, TILs detection method, TILs subsets and distribution site, the cut-off definition, follow-up period and outcome measures. The outcome measure of OS (overall survival), DFS (disease-free survival) or RFS (relapse-free survival) and odds ratio (OR) or hazard ratio (HR) as well as their associated 95\% confidence intervals $(95 \% \mathrm{Cl})$ were extracted for each incorporated study.

\section{Patients and tissue samples}

To validate the prognostic role of the TIL subset in gastric cancer, a cohort of 147 patients were diagnosed with gastric adenocarcinoma, and with complete 10 years follow-up data were recruited from the First Affiliated Hospital of Xi'an Jiao Tong University at the Department of Pathology. The median survival was 66 months, ranging from 3 to 144 months. The median age was 60 years; 116 patients were male, and 31 were female. The basic information of 147 cancer samples included in this study is shown in Table I. This study was approved by the local ethics committee. 
Table I. Patient characteristics $(n=147)$

\begin{tabular}{|c|c|}
\hline Feature & Frequency, $N(\%)$ \\
\hline \multicolumn{2}{|l|}{ Age [years]: } \\
\hline$\leq 45$ & $20(13.6)$ \\
\hline $45-60$ & $52(35.4)$ \\
\hline$\geq 60$ & $75(51.0)$ \\
\hline \multicolumn{2}{|l|}{ Sex: } \\
\hline Female & $31(21.1)$ \\
\hline Male & $116(78.9)$ \\
\hline \multicolumn{2}{|c|}{ Tumor location: } \\
\hline Cardia & $38(25.9)$ \\
\hline Antrum & $66(44.9)$ \\
\hline Corpus & $43(29.2)$ \\
\hline \multicolumn{2}{|c|}{ Lymph node involvement: } \\
\hline Negative & $62(42.2)$ \\
\hline Positive & $85(57.8)$ \\
\hline \multicolumn{2}{|l|}{ TNM stage: } \\
\hline$|A-| B$ & $39(26.5)$ \\
\hline$\|A-\| B$ & $40(27.2)$ \\
\hline$\|A-\| C$ & $68(46.3)$ \\
\hline IV & $0(0)$ \\
\hline \multicolumn{2}{|l|}{ Grade: } \\
\hline G1 & $3(2.0)$ \\
\hline G2 & $58(39.5)$ \\
\hline G3 & $70(47.6)$ \\
\hline G4 & $16(10.9)$ \\
\hline
\end{tabular}

\section{Immunohistochemistry}

Immunohistochemical staining was performed on paraffin-embedded cancer tissue sections that had been serially sectioned at 4-mm thickness. The sections were deparaffinized in xylene and rehydrated in descending concentrations of ethanol. Antigen retrieval was performed by heat-induced epitope retrieval methods in citrate buffer ( $\mathrm{pH}$ 6.0). Endogenous peroxidase activity was blocked by incubating in 3\% hydrogen peroxide in methanol for $15 \mathrm{~min}$. The sections were incubated for $2 \mathrm{~h}$ at room temperature (RT) with primary monoclonal antibodies: CD8 (DakoCytomation, Glostrup, Denmark; 1 : 100 dilution); granzyme B (Novocastra, Newcastle, UK; 1 : 100); OX40 (Novocastra; 1 : 30); FOXP3 (Abcam, Cambridge, UK; 1 : 50). Incubation with Dako EnVision (DakoCytomation, Denmark) for $30 \mathrm{~min}$ at RT, and followed by color development with DAB and counterstaining with hematoxylin. Surgical resected chronic inflammatory human tonsil tissue was used as the positive control. The negative control for immunostaining was prepared by incubating tissue sections without primary antibody. The positive TILS were counted by the eyepiece micrometer under the light microscope at 400× magnification. The density of TILS within the tumor specimens were evaluated as follows: (1) immune cells were counted in ten of the highest densities of TILs in different fields of each section; (2) the cells were counted in the intratumoral compartment and the necrotic areas were excluded; (3) two observers counted the cells at the same time, in the same field, using a multiple-lens microscope at 400x; (4) the results were expressed as the mean \pm standard error of the mean; (5) the densities of the positive TILs were grouped into TILshigh and TILs-low groups based on the median for further analysis.

\section{Statistical analysis}

Reading all the incorporated literature carefully; extracting and collating data according to the requirements of meta-analysis; creating data tables; and reviewing data to ensure its accuracy were performed. STATA version 12.0 (Stata Corporation, College Station, TX, USA) was used to perform the further analysis, with significance defined as a $p$-value less than 0.05 . The fixed-effects model or the random-effects model was considered according to heterogeneity, and the $I^{2}$ statistic was used to evaluate heterogeneity. If each independent study was homogeneous $\left(I^{2}<50 \%\right)$, the fixed-effect model was selected for analysis; if significant heterogeneity existed $\left(I^{2}>50 \%\right)$, the random effects model would be used to deal with it. The death risk of gastric cancer patients with different levels of TILs was evaluated by hazard ratios (HRs) and 95\% Cls. An observed HR $>1$ indicated worse prognosis in patients with high density TILs and a HR $<1$ suggested better prognosis. The publication bias was evaluated by visually as sessing the asymmetry of an inverted funnel plot, and was quantified by Begg's and Egger's tests. Survival rates of our verification samples were analyzed with the Kaplan-Meier method, and differences were compared with the log-rank test. Statistical analyses were performed with SPSS 13.0 for Windows (SPSS, Chicago, IL) and two-sided $p<0.05$ was considered statistically significant.

\section{Results}

Using the described searching strategy, a total of 995 records were identified in a primary literature search. After screening the titles, abstracts, publication types and full text of each publication, 955 studies were excluded, including reviews, small sample size articles, duplicate articles, animal studies and irrelevant studies. Only 40 studies investigated the correlation between TILs and patients' outcome in gastric cancer. However, 22 articles were excluded, due to lacking essential results concerning data extraction. Finally, 18 articles were include in the meta-analysis involving 
3020 cases. The flow diagram for study selection is shown in Figure 1.

\section{Study characteristics}

Table II summarized the characteristics of all included studies [14-31]. The summarizes should represented by summarized incorporated studies were published from 2003 to 2016 and conducted in China $(n=6)$, Germany $(n=1)$, Korea $(n=6)$, Japan $(n=3)$, America $(n=1)$, and Italy $(n=1)$. Among these, only 3 articles that reported the prognostic value of generalized TILs and TIL subsets were evaluated in 15 studies. Sample size of each study ranged from 52 to 487 patients; $H$ \& E (hematoxylin-eosin) staining and immunohistochemical (IHC) staining were applied for the detection of generalized TILs and specific TIL subsets respectively in the primary tumor tissue. The cut-off values contained the median level $(n=14)$, mean level $(n=1)$, and other semiquantitative methods $(n=3)$. HRs and $95 \% \mathrm{Cl}$ for overall survival (OS), disease-free survival (DFS), or recurrence-free survival (RFS) were extracted directly from the included studies. The assessment of publication bias for individual studies is presented in Table III, which showed that 18 studies were of high quality.

\section{The prognostic value of whole TILs in general}

Only three articles $[14,22,27]$ described the relation between the density of whole TILs and patients' survival. One of the articles evaluated the association of TILs in tumor nest with OS $(\mathrm{HR}=0.55,95 \% \mathrm{Cl}: 0.30-0.99, p=0.047)$ and DFS $(\mathrm{HR}=0.56,95 \% \mathrm{Cl}: 0.32-0.98, p=0.043)$, and one article assessed the relation between TILs in tumor stroma with RFS (HR = 12.193, 95\% Cl: $1.039-$ 143.055, $p=0.047)$ as well as DFS (HR $=4.836$, 95\% Cl: 0.917-25.525, $p=0.063)$. One article described the connection between the intratumoral TILS and $\mathrm{OS}(\mathrm{HR}=0.21,95 \% \mathrm{Cl}: 0.145-0.286, p=0.013)$. As the above results showed, we can approximately draw a conclusion that high density of whole TILS was related to a favorable prognosis in GC.

\section{Pooled analysis of TIL subsets}

Fifteen studies were assessed for the prognostic value of TIL subsets in GC patients.

\section{CD8+ lymphocytes}

There were 7 articles [15, 18, 20, 24, 27, 29, 31] investigating the prognostic value of intratumoral CD8+ T cells for OS. Our analysis results showed $P^{2}=4.3 \%$ and $p=0.394$, with a good homogeneity. The fixed-effects model was chosen to make a further analysis, and the results indicated that

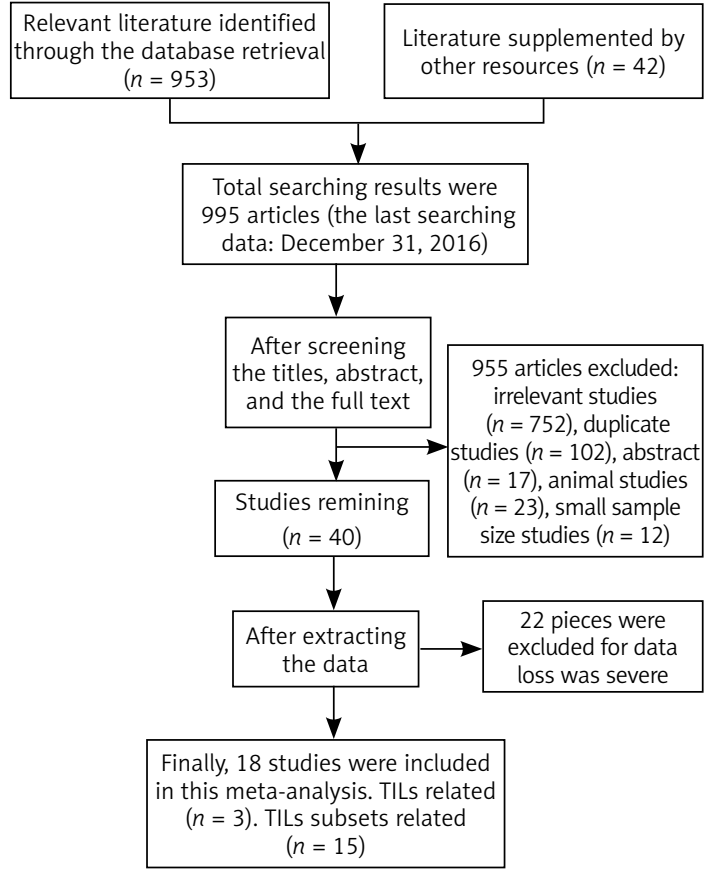

Figure 1. Flow diagram of the study selection process

a higher density of CD8+ T lymphocytes demonstrated a significantly longer OS $(H R=0.699$, $95 \% \mathrm{Cl}: 0.625-0.782, p<0.001$, Figure 2 A). Only 2 works [24, 31] studied the contact of CD8+ $T$ cells with DFS in intratumoral tissue, so we did not make the further analysis, due to its unreliable credibility. For the activated CTL (GrB+) cells, only one work [17] separately described the relationship between GrB+ TILs and DFS in tumor stromal tissue $(\mathrm{HR}=0.87,95 \% \mathrm{Cl}: 0.33-2.29, p=0.78)$ and tumor parenchyma $(\mathrm{HR}=0.72,95 \% \mathrm{Cl}: 0.21-$ 2.53, $p=0.61$ ). One work [20] discussed the effect of GrB + TILs in the intratumoral site on OS (HR = $0.828,95 \% \mathrm{Cl}: 0.500-1.369, p=0.461)$. It seems that GrB + T cells have no contribution to the prognosis of patients with gastric cancer.

\section{CD3+ T lymphocytes}

Eight papers $[15,17,20,24,25,27,29,30]$ investigated the prognostic value of CD3+ TILs in gastric cancer patients. Seven studies $[15,20,24,25$, $27,29,30]$ researched the connection of CD3+ TILS in the intratumoral site with OS, with a pooled $\mathrm{HR}$ of 0.610 for TILs-high cases (95\% Cl: 0.526-0.708, $p<0.001$ ), without any heterogeneity (Figure $2 \mathrm{~B}$ ). Only one study [17] reported the CD3+ TILs in tumor stroma (TS) and tumor parenchyma (TP), and the results showed that HRs for DFS were $0.86(95 \% \mathrm{Cl}$ : $0.33-2.23, p=0.76)$ and $0.76(95 \% \mathrm{Cl}: 0.29-1.98$, $p=0.58)$ respectively. Thus, it is reliable that a high level of intratumoral CD3+ TILS is associated with better outcome in GC. 


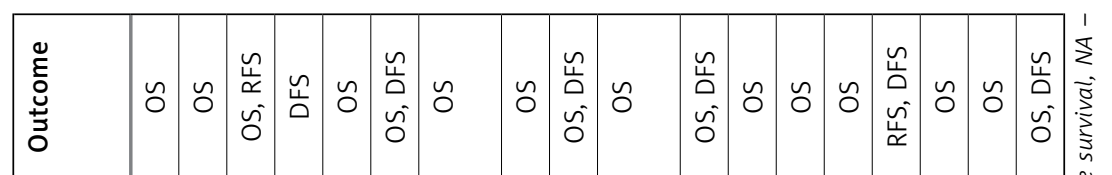

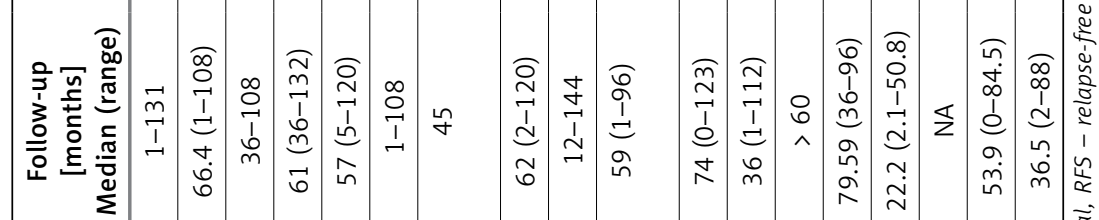

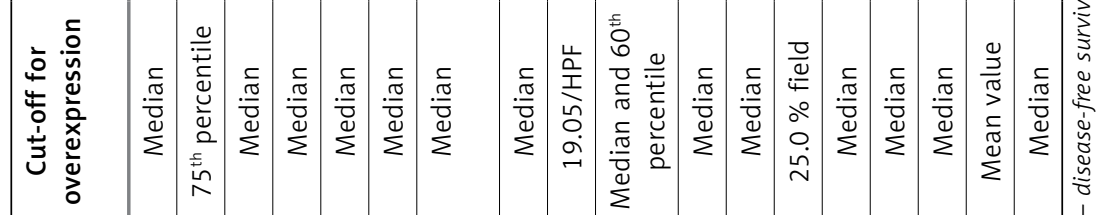

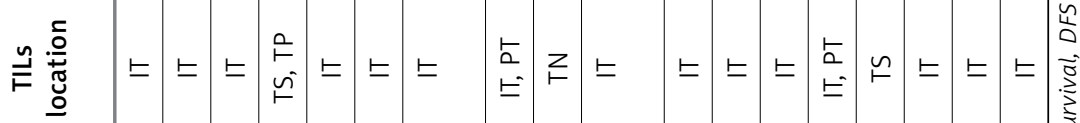

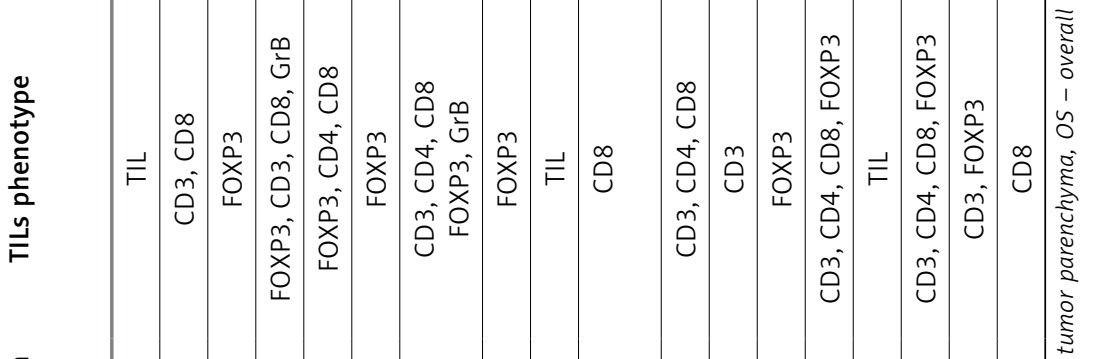

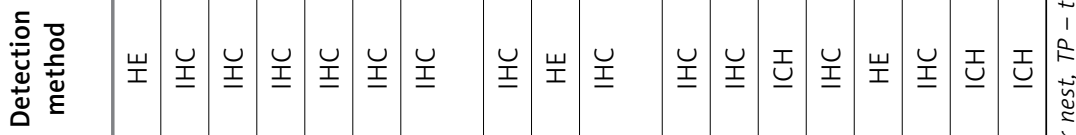

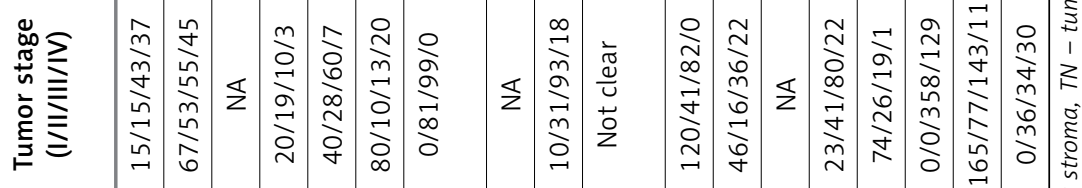
غे

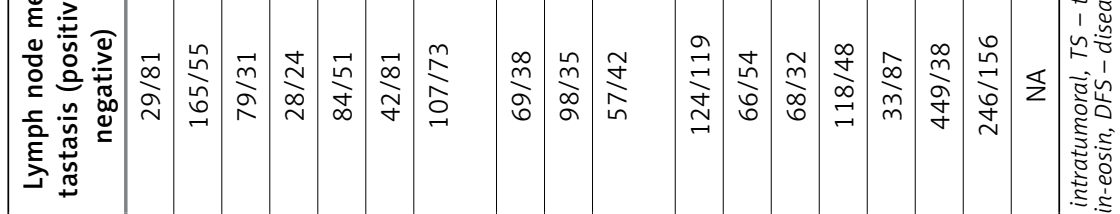

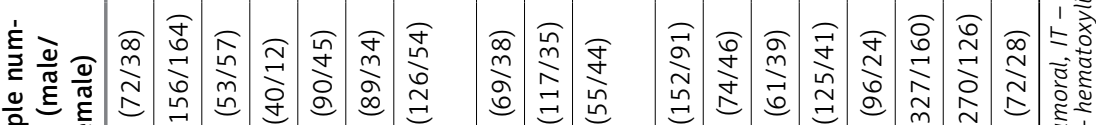

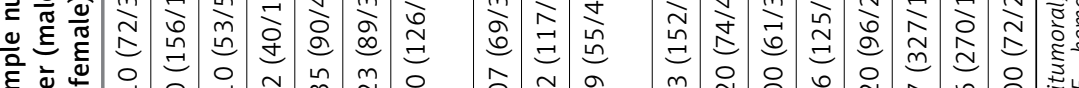

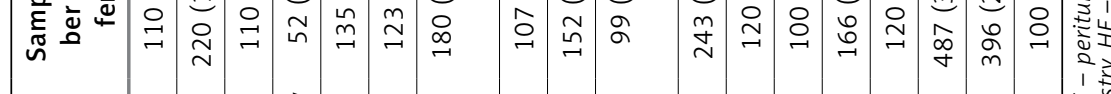

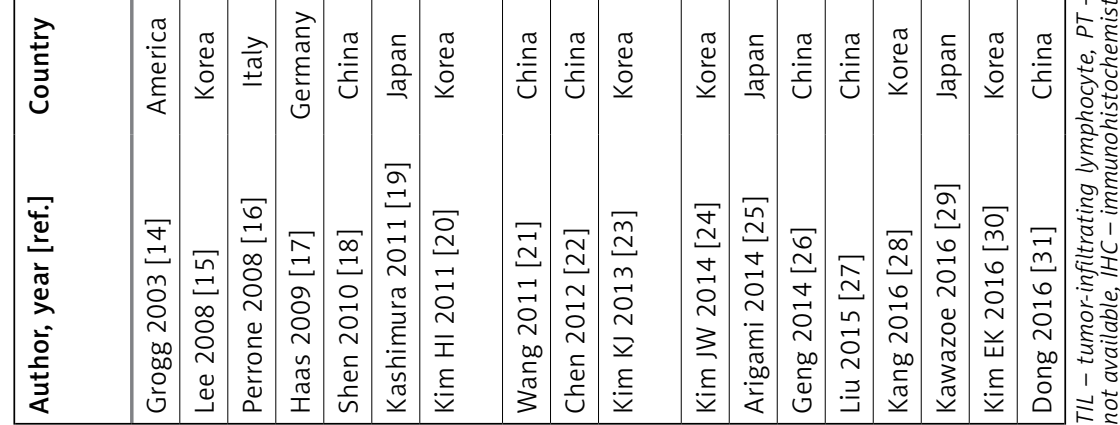


Table III. Pooled associations between intratumoral TIL subsets and overall survial of patients with gastric cancer

\begin{tabular}{|lccccccc|}
\hline Subsets & $\begin{array}{c}\text { No. of } \\
\text { studies }\end{array}$ & $\begin{array}{c}\text { No. of } \\
\text { patients }\end{array}$ & $\begin{array}{c}\text { Analysis } \\
\text { model }\end{array}$ & HR $(95 \% \mathrm{Cl})$ & $P$-value & $\begin{array}{c}\text { Heteroge- } \\
\text { neity } I^{2}, p\end{array}$ & $\begin{array}{c}\text { Publication bias } \\
\text { Begg's } p, \text { Egger's } p\end{array}$ \\
\hline CD8+ TIL & 7 & 1630 & Fixed & $\begin{array}{c}0.699 \\
(0.625-0.782)\end{array}$ & $<0.001$ & $4.3 \%, 0.394$ & $0.368,0.825$ \\
\hline CD4+ TIL & 5 & 1211 & Fixed & $\begin{array}{c}0.770 \\
(0.673-0.881)\end{array}$ & $<0.001$ & $\begin{array}{c}47.5 \%, \\
0.107\end{array}$ & $0.806,0.578$ \\
\hline CD3+ TIL & 7 & 1812 & Fixed & $\begin{array}{c}0.610 \\
(0.526-0.708)\end{array}$ & $<0.001$ & $0.0 \%, 0.926$ & $0.230,0.391$ \\
\hline FOXP3+ & 9 & 1793 & Random & $\begin{array}{c}1.382 \\
(0.944-2.025)\end{array}$ & 0.096 & $77.4 \%$, & $0.466,0.080$ \\
TIL & & & & & & & \\
\hline
\end{tabular}

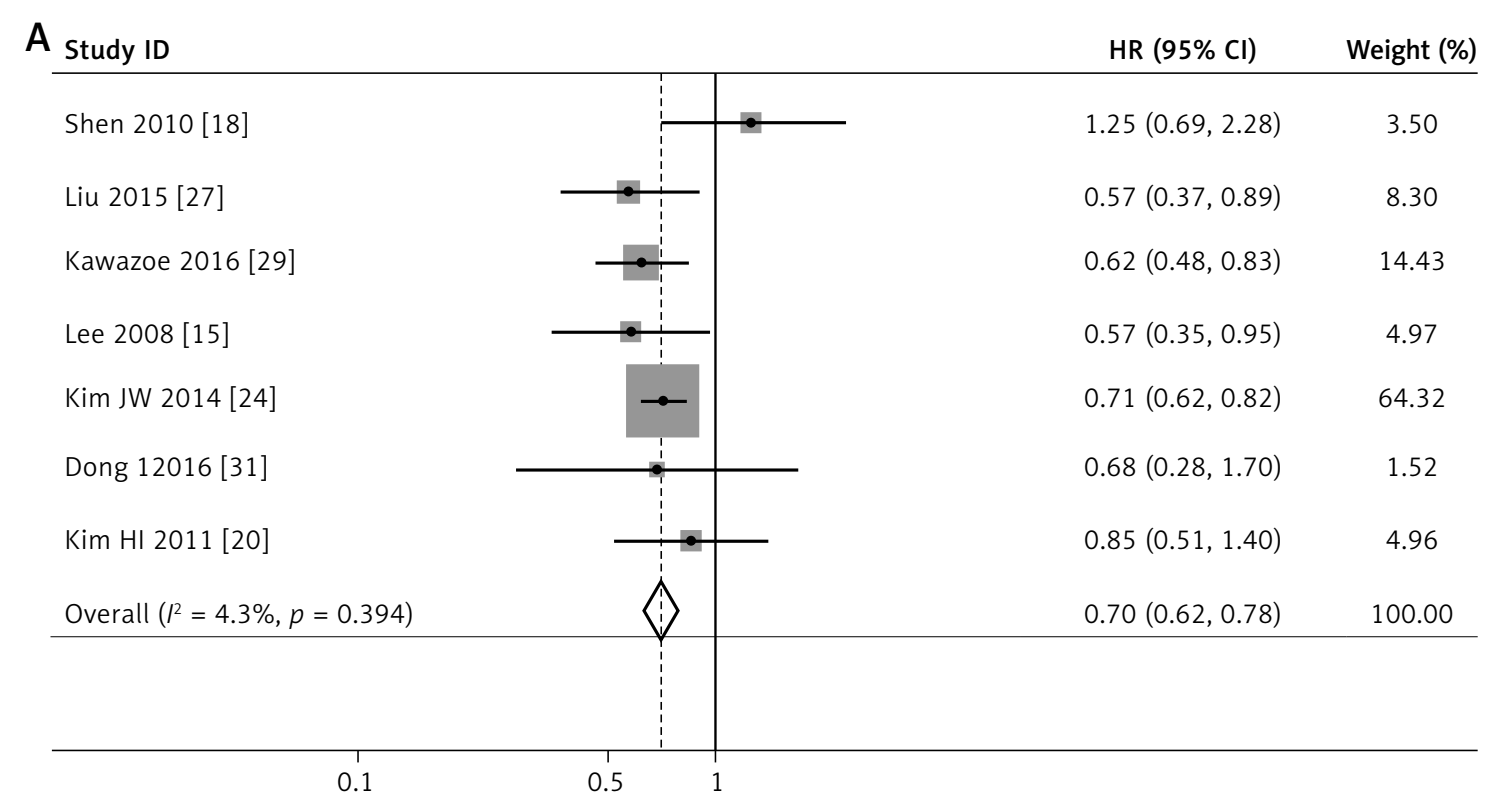

B Study ID

Liu 2015 [27]

Kawazoe 2016 [29]

Kim EK 2016 [30]

Lee 2008 [15]

Kim JW 2014 [24]

Arigami 2014 [25]

Kim HI 2011 [20]

Overall $\left(I^{2}=0.0 \%, p=0.928\right)$
$\mathrm{HR}(95 \% \mathrm{Cl})$

Weight (\%)

$\begin{array}{ll}0.49(0.31,0.78) & 10.54 \\ 0.69(0.51,0.92) & 25.47 \\ 0.60(0.40,1.00) & 10.56 \\ 0.55(0.32,0.95) & 7.34 \\ 0.58(0.43,0.78) & 25.00 \\ 0.65(0.42,0.97) & 12.65 \\ 0.65(0.39,1.09) & 8.43 \\ 0.61(0.53,0.71) & 100.00\end{array}$

Figure 2. Forest plots of studies evaluating hazard ratios of high level of intratumoral TIL subsets in gastric cancer. Forest plots of the prognostic effect on OS of CD8+ T cells (A), CD3+ T cells (B) 


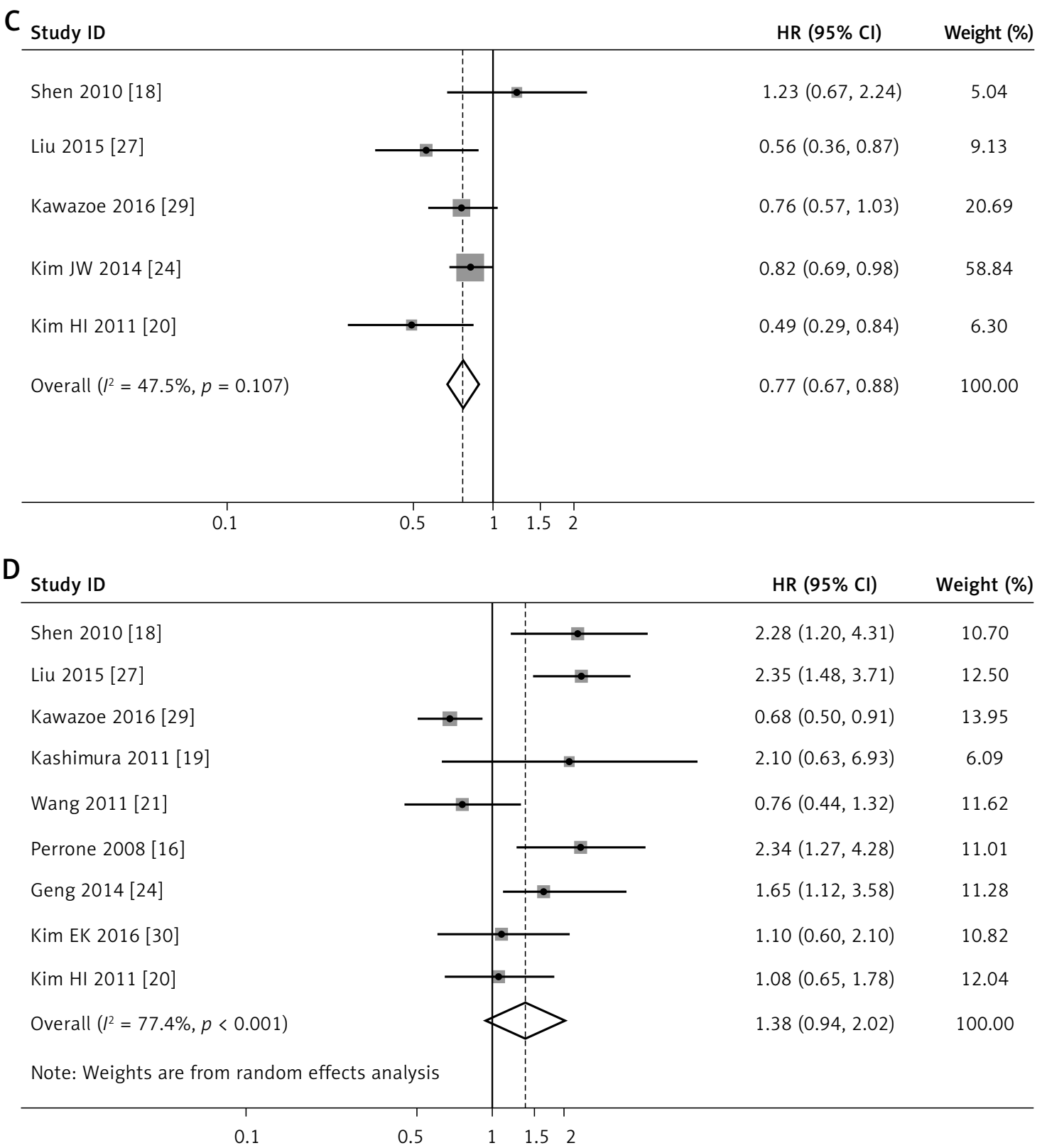

Figure 2. Cont. Forest plots of studies evaluating hazard ratios of high level of intratumoral TIL subsets in gastric cancer. Forest plots of the prognostic effect on OS of CD4+ T cells (C), FOXP3+ T cells (D)

\section{CD4+ T lymphocyte}

For CD4+ TILs, we found a pooled HR of 0.770 (95\% Cl: $0.673-0.881, p<0.001$, Figure 2 C) using 5 incorporated studies [18, 20, 24, 27, 29], indicating that a higher CD4+ TILs level in the intratumoral region significantly decreased risk of death for GC patients. Heterogeneity between studies remained not obvious with $R^{2}=47.5 \%$.

\section{FOXP3+ T lymphocyte}

Nine studies [16, 18, 19, 20, 21, 26, 27, 29, 30] investigated the effect of intratumoral FOXP3+ TILS on OS, with a pooled HR of 1.382 with a $95 \% \mathrm{Cl}$ of
0.944-2.025, not reaching statistical significance in this meta-analysis $(p=0.096)$. A random effects model was used due to the high heterogeneity noted between studies $\left(R^{2}=77.4 \%\right.$, Figure $\left.2 \mathrm{D}\right)$.

\section{Publication bias}

Begg's and Egger's test were conducted to assess the publication bias of all included studies. The $p$ values for Begg's test were 0.230-0.806, and for Egger's test were 0.080-0.825. Additionally, a funnel plot was applied to detect publication bias for the FOXP3+ TILs subgroup, with the largest number of studies. The funnel plot of the in- 
tratumoral FOXP3+ T cell infiltration was substantially symmetric (Figure 3 ). Thus, the results above suggest that publication bias was not evident in this meta-analysis (Table III).

\section{Validation of the prognostic value of intratumoral TILs in primary gastric tumor tissue}

To validate the prognostic role of the TIL subset in gastric cancer, we did a similar experiment to validate the meta-analysis results. The immune markers we chose were not completely the same as it was mentioned in the meta-analysis. CD3 molecules are expressed on all T cells and functionally $T$ cells are classified as cytotoxic $T$ cells (CD8+) and helper T cells (CD4+). CD8+ T cells are well known to be designated CTLs, as for the CD4+ $\mathrm{T}$ helper cells, OX40 are used for identifying the primed CD4+ T cells. The OX40 has induced expression after activation, and is mainly expressed on the activated CD4+ T cells, inducing cytokine production and maintaining a normal immune response. So in our experiment, in addition to considering the quantity of $T$ cells infiltrating in the tumor microenvironment, the functional status was taken into consideration too. Consequently, we selected OX40 (expressing on the primed CD4+ T cells), granzyme B (expressing on the activated $C D 8+T$ cells), and FOXP3 as well as CD8, the

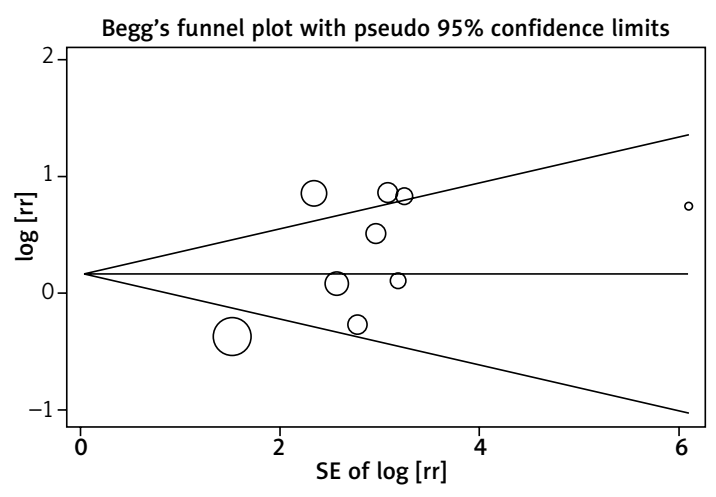

Figure 3. Funnel plots of association of intratumoral FOXP3+ T cell infiltration with overall survival in select studies

same marker with the meta-analysis. CD8+ and OX40+ cells were browned in the cell membrane; the brownish positive particles of GrB were located in the cytoplasm; and the FOXP3+ T cells are colored brown in nuclei (Figure 4). Our experimental results showed that there was no obvious correlation between the intratumoral CD8+, OX40+ as well as FOXP3+ TILS and the overall survival rate respectively, while a higher density of $\mathrm{GrB}+$ $T$ cell infiltration indicated an optimistic prognosis $(p=0.007$, Figure $5 \mathrm{~A})$. Regardless of the subsets of infiltrating lymphocytes, the density of whole TIL suggested a favorable prognosis ( $p=0.009$, Figure 5 B).
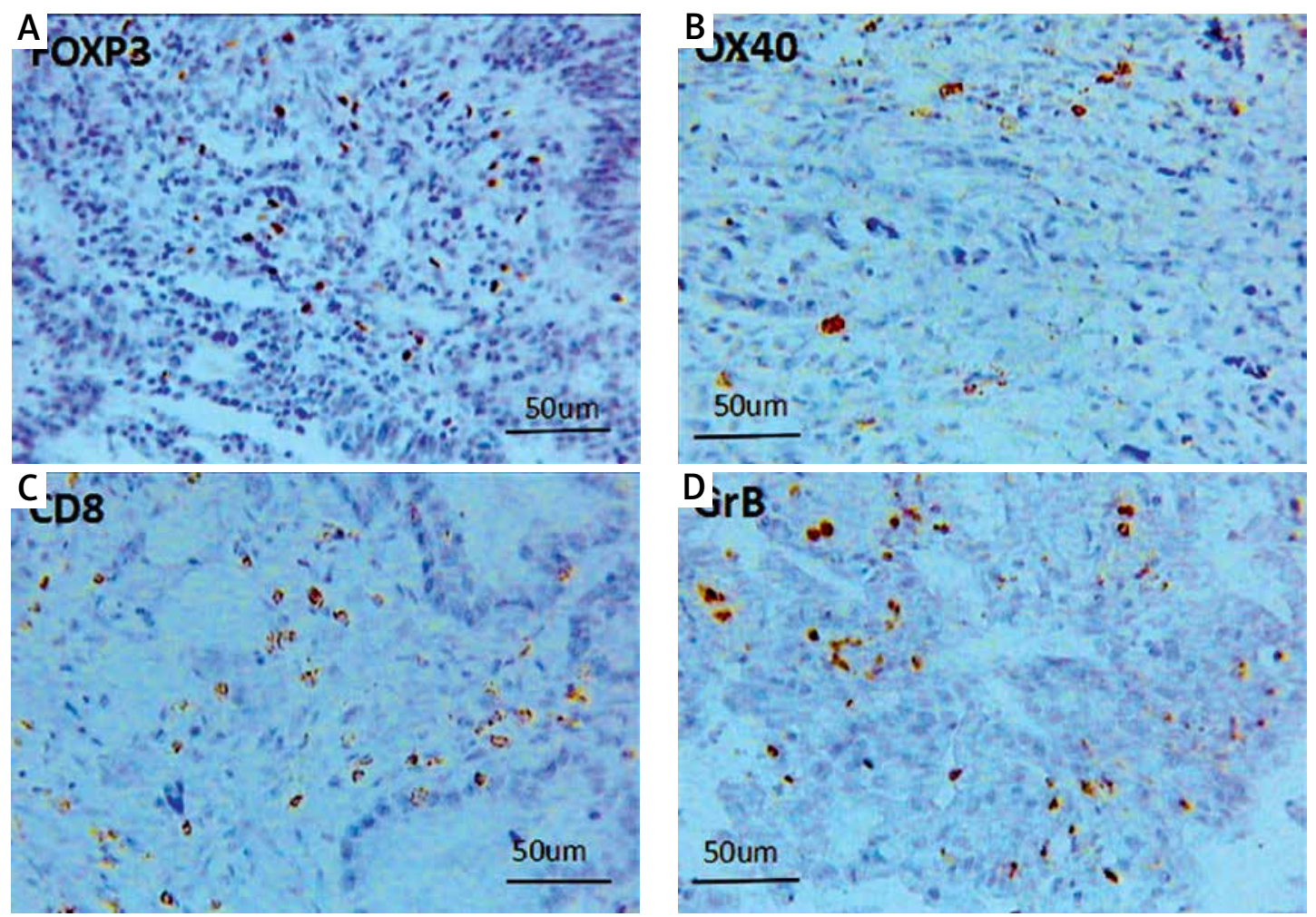

Figure 4. Images (400x) were the positive T cells of FOXP3 (A), OX40 (B), CD8 (C), and GrB (D), infiltrating intratumoral tissue 


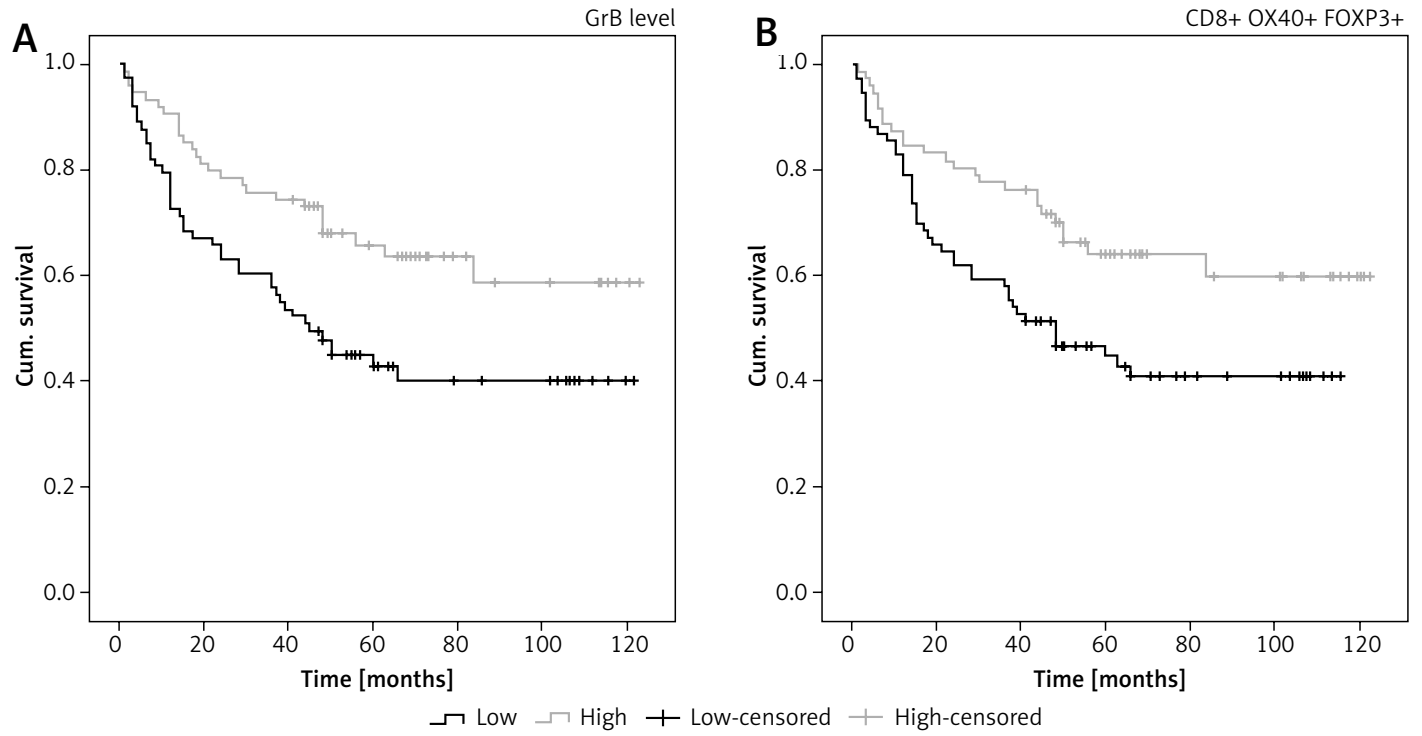

Figure 5. Kaplan-Meier curves. A, B - the survival rate with respect to the density of GrB+T cells $(p=0.007)$, and the total number of CD8+, FOXP3+, OX40+ T cells $(p=0.009)$

\section{Discussion}

The gastric tumor microenvironment is frequently filled with a wide range of immune cells, which have been reported to impact on cancer development, progression, and cancer-related immune reactions, emerging as the hotspot of cancer research [32, 33]. A large number of studies have shown that tumor infiltrating immune cells are closely related to the patients' prognosis, tumor recurrence and metastasis [34-36]. Due to variance in study design, sample size, and detection site, the direct impact of the TIL status on patient survival remains inconclusive and contradictory. Therefore, the TILs could not be used for grading or staging in clinics. This meta-analysis incorporated 18 studies involving 3020 patients aiming to systematically review the prognostic value of TILs in gastric cancer. Additionally, we also performed a similar study to validate the analysis results. To our limited knowledge, this is the first experimental demonstration with meta-analysis to discuss the prognostic values of various kinds of immune cells in GC. The results from the 3 included studies and our investigation confirmed that high density of TILs was associated with a favorable prognosis. Simultaneously, analysis of the TIL subsets including CD8+, GrB+, CD3+, CD4+ and FOXP3+ T cells was performed too. CD8+ lymphocytes are the main effective cells in the immune response, and a better OS in patients with high level of intratumoral CD8+ T cell infiltration was found in the results of this meta-analysis. However, CD8+ T cells did not reveal a positive prognostic value in our validation study, which may, on the one hand, be due to the limited samples, and on the other hand, be due to the complexity and multiplicity of CD8+ T cell function. CD8+ Treg cells have drawn more attention recently, such as the CD8+ CD28- T cells infiltrating human cancer which could inhibit both $T$ cell proliferation and cytotoxicity [37]. Tumor-infiltrating CD8+ T cells with high CD39 expression exhibited features of exhaustion, and the exhausted CD39+CD8+ $T$ cells were present within tumors and invaded or metastatic lymph nodes, but were barely detectable within noninvaded lymph nodes, and inhibited IFN- $\gamma$ production by responder $\mathrm{CD} 8+\mathrm{T}$ cells [38]. Additionally, there are many distinct states of CD8+ T cell dysfunction in tumors, such as $T$ cell tolerance to self-antigens and tumor-induced T cell dysfunction [39]. Genome-wide transcriptional analysis has revealed self-tolerant CD8+ $T$ cells to harbor a tolerance-specific gene program, with hundreds of genes differentially expressed compared to their naive or memory counterparts [40]. Comprehensive analysis of CD8+ T cell status are needed imperatively. Research for activated CD8 $+T$ cells with the presence of GrB is limited, and the results from other studies did not reveal a positive prognostic value. Due to $\mathrm{GrB}+\mathrm{T}$ cells playing a main role of the cytotoxic effect, we added the GrB+ lymphocytes to our experiment, and the result showed a better outcome with higher density of intratumoral GrB+ T cell infiltration ( $p=0.007$, Figure $5 \mathrm{~A}$ ). As for the CD4 cells, a high density of intatumoral CD4+ T cell infiltration would decrease the death risk in our meta-analysis results. In our validating experiment, we took OX40 phenotype to denote the CD4 T cells, but it did not indicate better prognostic value as CD4+ $T$ cells. It is known that CD4+ T cells are essential organizers of cell-mediated immunity, participat- 
ing in each stage of the immune response. Naive CD4+ $T$ cells can be induced to differentiate to specific lineages according to the local cytokine milieu, towards Th1, Th2, Th17 and Treg [41]. And Th17 cells play a potent pro-inflammatory role in certain infections, tumor and autoimmune disorders [42, 43]. The complicated role of Th17 cells in tumors tissue was well reviewed by Qi et al. [44], and Th17 cells provided functions of both antitumoral and protumoral activity in different cancers with different stage. The author pointed out that the tumor microenvironment could affect Th17 cells' developmental plasticity, which can convert into other subsets of T cells, including Th1 cells and Tregs. Thus, future studies need to focus on defining the molecular mechanisms and identifying the tumor microenvironment factors responsible for the function of Th17 cells in tumor immunity. For the much more heterogeneous CD4+ T cell population, more research is needed.

A high density of FOXP3 $+\mathrm{T}$ cell infiltration may increase the death risk in GC $[16,18]$. However, one study [17] found that FOXP3 + T cells indicated better progression-free survival, while another study [20] found they had no prognostic value, and our results of the analysis revealed that FOXP3+ lymphocytes may not indicate the patients' prognosis. These results suggested a complex role of FOXP3+ lymphocytes for GC, which may result from the intricate function of FOXP3+ $T$ cells with respect to their surface phenotypes, cell subsets, and functional heterogeneity [45]. The FOXP3+ T cells are known as a subpopulation of $T$ cells which modulate the immune system, with immunosuppressive activities by producing anti-inflammatory cytokines, such as IL-10 and TGF- $\beta$ [46], and related to poor prognosis in many cancers [47]. However, in some types of cancer, a high level of FOXP3+ T cells was associated with a positive prognosis, such as colorectal carcinoma and follicular lymphoma. This could be due to Tregs' ability to suppress general inflammation, which is known to trigger cell proliferation and metastasis $[48,49]$. These results suggested that FOXP3+ lymphocytes' role in the development of cancer is highly dependent on both type and location of the tumor. Consequently, the molecular mechanisms governing Treg recruitment and function in cancer are urgently required to be understood in more detail in individual tumor types. T cells with CD3 positivity, a pan T cell marker, were found associated with better OS by the integrated analysis of the seven studies. Our data showing that the density of whole TILs indicated a favorable prognosis were in line with the above-mentioned results. A study in colon cancer revealed that type, density, and location of immune cells within human colorectal tumors could predict clin- ical outcome [50], and many other reports favor the better indicator of $\mathrm{CD} 3+\mathrm{T}$ cell infiltrates in digestive tracts [51-53]. A meta-analysis of $T$ cell infiltrates in ovarian cancer [54] found that higher CD3+ lymphocyte levels were a worse prognostic marker, for which further validation is needed because of insufficient studies included.

The present meta-analysis results revealed that various intratumoral TIL subsets were indeed associated with outcome of patients with GC, and the pooled HRs showed dramatic statistical significance. The publication bias detected by Begg's and Egger's test showed less significance and the conclusion was reliable. However, the results from our study should be interpreted with caution because we might have failed to identify some published and unpublished studies with negative results or with limited data that would have affected our pooled estimates. We presumed that the potential sources of bias were as follows: (1) Different cut-off values could result in bias. In our meta-analysis, the median cutoff values were mainly used, and there were still 4 studies that used other methods. (2) Not all of the incorporated studies provided a median follow-up time, so the results were uncertain. (3) The number of included documents was too small; and the research objects were of different race and different region. (4) Although all studies have used immunohistochemical methods, the discrepancy in tissue fixation, antibodies used for T cell detection and other operational inconsistencies may lead to inevitable bias. Although there are some limitations in this meta-analysis, it is possible that the density of lymphocyte infiltration may be a reliable predictor for prognosis in gastric cancer patients. Global multi-center collaborating investigation [55] have been carried out on colorectal carcinoma by Immunoscore by which the infiltrating CD3+ and CD8+ $T$ cells within the cancer center and cancer margin were extensively calculated by digital pathology technique. This initiative may pave the way to utilize tumor infiltrating lymphocytes as a predictive marker for oncology clinical practice in the future.

In conclusion, this meta-analysis suggests that TILs were prognostic markers for OS in GC patients to a large degree. In addition, a high density of intratumoral CD8+, CD3+, and CD4+ lymphocyte indicated good prognosis in GC, while FOXP3+ lymphocytes demonstrated no obvious effect on survival outcomes. However, due to the limitations of this study, TILs cannot fully explain their impact on prognosis. Therefore, further studies should focus on high-quality prospective studies, including a comprehensive clinical pathology, evaluation of information, follow-up strategy, and standardized cut-off values, similar treatment strategies, and multivariate analyses of clinicopathological variables of the pa- 
tients, which will make the study more standardized. Furthermore, quantitative studies of TILs alone are far from explaining the complex effects of the tumor microenvironment, requiring more rigorous design, larger sample size, more standardized survival analyses, and longer follow-up studies to produce more credible statistics.

\section{Conflict of interest}

The authors declare no conflict of interest.

\section{References}

1. McCarty M. Principles of prognosis in cancer. J Am Med Assoc 1931; 96: 30-33.

2. Gooden MJ, de Bock GH, Leffers N, Daemen T, Nijman HW. The prognostic influence of tumour-infiltrating lymphocytes in cancer: a systematic review with meta-analysis. Br J Cancer 2011; 105: 93-103.

3. Schreiber RD, Old LJ, Smyth MJ. Cancer immunoediting: integrating immunity's roles in cancer suppression and promotion. Science 2011; 331: 1565-70.

4. Peker KD, Ozkanli SS, Akyuz C, et al. Preoperative immunonutrition regulates tumor infiltrative lymphocytes and increases tumor angiogenesis in gastric cancer patients. Arch Med Sci 2017; 13: 1365-72.

5. Balch CM, Riley LB, Bae YJ, et al. Patterns of human tumor-infiltrating lymphocytes in 120 human cancers. Arch Surg 1990; 125: 200-5.

6. Al-Attar A, Shehata M, Durrant L, Moseley P, Deen S, Chan S. T cell density and location can influence the prognosis of ovarian cancer. Pathol Oncol Res 2010; 16: 361-70.

7. Al-Shibli KI, Donnem T, Al-Saad S, Persson M, Bremnes RM, Busund LT. Prognostic effect of epithelial and stromal lymphocyte infiltration in non-small cell lung cancer. Clin Cancer Res 2008; 14: 5220-7.

8. Cho Y, Miyamoto M, Kato K, et al. CD4+ and CD8+ T cells cooperate to improve prognosis of patients with esophageal squamous cell carcinoma. Cancer Res 2003 63: 1555-9.

9. Mizukami Y, Kono K, Kawaguchi Y, et al. Localisation pattern of Foxp3+ regulatory $T$ cells is associated with clinical behaviour in gastric cancer. Br J Cancer 2008; 98: 148-53.

10. Ichihara F, Kono K, Takahashi A, Kawaida H, Sugai H, Fujii $\mathrm{H}$. Increased populations of regulatory $T$ cells in peripheral blood and tumor-infiltrating lymphocytes in patients with gastric and esophageal cancers. Clin Cancer Res 2003; 9: 4404-8.

11. Ciebiada M, Kasztalska K, Gorska-Ciebiada M, Barylski M, Gorski P. Expression of IL-7 receptor in human peripheral regulatory T cells. Arch Med Sci 2013; 9: 555-60.

12. El-Maraghy N, Ghaly MS, Dessouki O, Nasef SI, Metwally L. CD4+CD25-Foxp3+ T cells as a marker of disease activity and organ damage in systemic lupus erythematosus patients. Arch Med Sci 2018; 14: 1033-40.

13. Jerzyńska J, Stelmach W, Rychlik B, et al. Clinical and immunological effects of vitamin $D$ supplementation during the pollen season in children with allergic rhinitis. Arch Med Sci 2018; 14: 122-31.

14. Grogg KL, Lohse CM, Pankratz VS, Halling KC, Smyrk TC. Lymphocyte-rich gastric cancer: associations with Epstein-Barr virus, microsatellite instability, histology, and survival. Mod Pathol 2003; 16: 641-51.
15. Lee HE, Chae SW, Lee YJ, et al. Prognostic implications of type and density of tumour-infiltrating lymphocytes in gastric cancer. Br J Cancer 2008; 99: 1704-11.

16. Perrone G, Ruffini PA, Catalano V, et al. Intratumoural FOXP3-positive regulatory $T$ cells are associated with adverse prognosis in radically resected gastric cancer. Eur J Cancer 2008; 44: 1875-82.

17. Haas M, Dimmler A, Hohenberger W, Grabenbauer GG, Niedobitek G, Distel LV. Stromal regulatory T-cells are associated with a favourable prognosis in gastric cancer of the cardia. BMC Gastroenterol 2009; 9: 65.

18. Shen Z, Zhou S, Wang Y, et al. Higher intratumoral infiltrated Foxp3+ Treg numbers and Foxp3+/CD8+ ratio are associated with adverse prognosis in resectable gastric cancer. J Cancer Res Clin Oncol 2010; 136: 1585-95.

19. Kashimura S, Saze Z, Terashima M, et al. CD83(+) dendritic cells and Foxp3(+) regulatory T cells in primary lesions and regional lymph nodes are inversely correlated with prognosis of gastric cancer. Gastric Cancer 2012; 15: 144-53.

20. Kim HI, Kim H, Cho HW, et al. The ratio of intra-tumoral regulatory T cells(Foxp3+)/helper T cells (CD4+) is a prognostic factor and associated with recurrence pattern in gastric cardia cancer. J Surg Oncol 2011; 104: 728-33.

21. Wang B, Xu D, Yu X, et al. Association of intra-tumoral infiltrating macrophages and regulatory $T$ cells is an independent prognostic factor in gastric cancer after radical resection. Ann Surg Oncol 2011; 18: 2585-93.

22. Chen LJ, Zheng X, Shen YP, et al. Higher numbers of T-bet(+) intratumoral lymphoid cells correlate with better survival in gastric cancer. Cancer Immunol Immunother 2013; 62: 553-61.

23. Kim KJ, Lee KS, Cho HJ, et al. Prognostic implications of tumor-infiltrating FoxP3+ regulatory $T$ cells and CD8+ cytotoxic T cells in microsatellite-unstable gastric cancers. Hum Pathol 2014; 45: 285-93.

24. Kim JW, Nam KH, Ahn SH, et al. Prognostic implications of immunosuppressive protein expression in tumors as well as immune cell infiltration within the tumor microenvironment in gastric cancer. Gastric Cancer 2016; 19: 42-52.

25. Arigami T, Uenosono Y, Ishigami S, et al. Decreased density of CD3+ tumor-infiltrating lymphocytes during gastric cancer progression. J Gastroenterol Hepatol 2014; 29: 1435-41.

26. Geng Y, Wang H, Lu C, et al. Expression of costimulatory molecules B7-H1, B7-H4 and Foxp3+ Tregs in gastric cancer and its clinical significance. Int J Clin Oncol 2015; 20: 273-81.

27. Liu K, Yang K, Wu B, et al. Tumor-infiltrating immune cells are associated with prognosis of gastric cancer. Medicine (Baltimore) 2015; 94: e1631.

28. Kang BW, Seo AN, Yoon S, et al. Prognostic value of tumor-infiltrating lymphocytes in Epstein-Barr virus-associated gastric cancer. Ann Oncol 2016; 27: 494-501.

29. Kawazoe A, Kuwata T, Kuboki Y, et al. Clinicopathological features of programmed death ligand 1 expression with tumor-infiltrating lymphocyte, mismatch repair, and Epstein-Barr virus status in a large cohort of gastric cancer patients. Gastric Cancer 2016; 20: 407-15.

30. Kim EK, Yoon SO, Jung WY, et al. Implications of NOVA1 suppression within the microenvironment of gastric cancer: association with immune cell dysregulation. Gastric Cancer 2017; 20: 438-47.

31. Dong J, Li J, Liu S, et al. Prognostic potential of an immune score based on the density of CD8+ T cells, CD20+ 
B cells, and CD33+/p-STAT1+ double-positive cells and HMGB1 expression within cancer nests in stage IIIA gastric cancer patients. Chin J Cancer Res 2016; 28: 543-52.

32. Lee K, Hwang H, Nam KT. Immune response and the tumor microenvironment: how they communicate to regulate gastric cancer. Gut Liver 2014; 8: 131-9.

33. Gurzu S, Orlowska J, Sugimura H, et al. Immunohistochemical features and staging of early gastric cancer Arch Med Sci 2017; 13: 1373-82.

34. García-Martínez E, Gil GL, Benito AC, et al. Tumor-infiltrating immune cell profiles and their change after neoadjuvant chemotherapy predict response and prognosis of breast cancer. Breast Cancer Res 2014; 16: 488.

35. Bremnes RM, Al-Shibli K, Donnem T, et al. The role of tumor-infiltrating immune cells and chronic inflammation at the tumor site on cancer development, progression, and prognosis: emphasis on non-small cell lung cancer. J Thorac Oncol 2011; 6: 824-33.

36. Forero A, Li Y, Chen D, et al. Expression of the MHC class II pathway in triple-negative breast cancer tumor cells is associated with a good prognosis and infiltrating lymphocytes. Cancer Immunol Res 2016; 4: 390-9.

37. Filaci G, Fenoglio D, Fravega M, et al. CD8+ CD28- T regulatory lymphocytes inhibiting $T$ cell proliferative and cytotoxic functions infiltrate human cancers. J Immunol 2007; 179: 4323-34.

38. Canale FP, Ramello MC, Núñez N, et al. CD39 Expression defines cell exhaustion in tumor-infiltrating CD8+ T cells. Cancer Res 2018; 78: 115-28.

39. Schietinger A, Greenberg PD. Tolerance and exhaustion: defining mechanisms of T cell dysfunction. Trends Immunol 2014; 35: 51-60.

40. Schietinger A, Delrow JJ, Basom RS, et al. Rescued tolerant CD8 T cells are preprogrammed to reestablish the tolerant state. Science 2012; 335: 723-7.

41. Middleton GW, Annels NE, Pandha HS. Are we ready to start studies of Th17 cell manipulation as a therapy for cancer? Cancer Immunol Immunother 2012; 61: 1-7.

42. Murdaca G, Colombo BM, Puppo F. The role of Th17 lymphocytes in the autoimmune and chronic inflammatory diseases. Intern Emerg Med 2011; 6: 487-95.

43. Ye J, Livergood RS, Peng G. The role and regulation of human Th17 cells in tumor immunity. Am J Pathol 2013; 182: $10-20$

44. Qi W, Huang X, Wang J. Correlation between Th17 cells and tumor microenvironment. Cell Immunol 2013; 285: 18-22.

45. Zhuo C, Xu Y, Ying $M$, et al. FOXP3+ Tregs: heterogeneous phenotypes and conflicting impacts on survival outcomes in patients with colorectal cancer. Immunol Res 2015; 61: 338-47.

46. Shevach EM. Mechanisms of FoxP3+T regulatory cellmediated suppression. Immunity 2009; 30: 636-45.

47. Whiteside TL. What are regulatory T cells (Treg) regulating in cancer and why? Semin Cancer Biol 2012; 22: 327-34.

48. Zhang X, Kelaria S, Kerstetter J, et al. The functional and prognostic implications of regulatory $T$ cells in colorectal carcinoma. J Gastrointest Oncol 2015; 6: 307-13.

49. Carreras J, Lopez-Guillermo A, Fox BC, et al. High numbers of tumor-infiltrating FOXP3-positive regulatory T cells are associated with improved overall survival in follicular lymphoma. Blood 2006; 108: 2957-64.

50. Galon J, Costes A, Sanchez-Cabo F, et al. Type, density, and location of immune cells within human colorectal tumors predict clinical outcome. Science 2006; 313: 1960-4.

51. Stein AV, Dislich B, Blank A, et al. High intratumoural but not peritumoural inflammatory host response is associated with better prognosis in primary resected oesophageal adenocarcinomas. Pathology 2017; 49: 30-7.

52. Deschoolmeester V, Baay M, Van Marck E, et al. Tumor infiltrating lymphocytes: an intriguing player in the survival of colorectal cancer patients. BMC Immunol 2010; 11: 19.

53. Turksma AW, Coupé VM, Shamier MC, et al. Extent and location of tumor-infiltrating lymphocytes in microsatellite-stable colon cancer predict outcome to adjuvant active specific immunotherapy. Clin Cancer Res 2016; 22: 346-56.

54. Hwang WT, Adams SF, Tahirovic E, et al. Prognostic significance of tumor-infiltrating $T$ cells in ovarian cancer: a meta-analysis. Gynecol Oncol 2012; 124: 192-8.

55. Galon J, Pagès F, Marincola FM, el at. Cancer classification using the Immunoscore: a worldwide task force. J Transl Med 2012; 10: 205. 